\title{
Does the new International Diabetes Federation definition of the metabolic syndrome predict CHD any more strongly than older definitions? Findings from the British Women's Heart and Health Study
}

Received: 13 July 2005 / Accepted: 11 August 2005 / Published online: 23 December 2005

C) Springer-Verlag 2005

\begin{abstract}
Aims/hypothesis: We compared the associations between three definitions of the metabolic syndrome and CHD risk. The definitions studied were the new International Diabetes Federation (IDF) definition, and those of the World Health Organization (WHO) and the National Cholesterol Education Programme (NCEP). Our aim was to determine whether the magnitudes of the associations for any of the syndrome definitions are greater than for the individual components. Materials and methods: A prospective cohort study of a random sample of 3,589 British women who were aged 60-79 years and free of CHD at baseline was performed. Among these women there were 194 incident cases of CHD (40 of them fatal) during 15,778 woman-years of follow-up. Results: Insulin resistance (homeostasis model assessment), triglyceride levels, systolic blood pressure, waist and waist-to-hip ratio were positively and linearly associated with CHD risk; HDL cholesterol was inversely associated with risk. All three definitions of the metabolic syndrome were modestly and similarly (to each other) associated with CHD risk. The ageadjusted hazard ratio $(95 \% \mathrm{CI})$ was $1.32(1.03,1.70)$ for IDF syndrome, $1.45(1.00,2.10)$ for WHO syndrome, and $1.38(1.00,1.93)$ for NCEP syndrome. Adjustment for smoking, inactivity and life-course socioeconomic position resulted in attenuation of these associations to $1.25(0.96$, $1.61), 1.31(0.90,1.90)$ and $1.27(0.90,1.79)$, respectively. The magnitudes of the associations for individual components of the syndrome were similar to those for any of the syndrome definitions. Conclusions/interpretation: The metabolic syndrome, defined by any of the three methods, is only modestly associated with CHD risk in this study of older women. Life-course socioeconomic position appears to be an important confounder in the association of the metabolic syndrome with CHD risk.
\end{abstract}

D. A. Lawlor $(\bowtie) \cdot$ G. D. Smith · S. Ebrahim

Department of Social Medicine, University of Bristol,

Bristol, UK

e-mail: d.a.lawlor@bristol.ac.uk

Tel.: +44-117-9287267

Fax: +44-117-9287325
Keywords Coronary heart disease $\cdot$ Epidemiology

Insulin resistance $\cdot$ Metabolic syndrome

Abbreviations HOMA: homeostasis model assessment . IDF: International Diabetes Federation - NCEP:

National Cholesterol Education Programme - WHO:

World Health Organization

\section{Introduction}

A recent systematic review and meta-analysis concluded that the metabolic syndrome, as defined by the two most widely used definitions (the World Health Organization [WHO] and the US National Cholesterol Educational Program [NCEP] definitions [text box]), did only a 'modest job' of predicting cardiovascular disease (estimated summary relative risk of 1.7 to 1.9 ) [1]. Notably, that review found that the effects published in the earlier studies (which have been widely cited and used to suggest that the metabolic syndrome is a major CHD and cardiovascular disease risk factor) were considerably greater than those published in later studies [1].

One explanation for the weaker association in some studies compared to others is the difference in confounding factors that have been taken into account in different studies. Although several studies have demonstrated independent associations between both childhood and adulthood socioeconomic position, and components of the metabolic syndrome $[2,3]$ and CHD risk $[4,5]$, no previous study has, to our knowledge, taken account of the effect of socioeconomic position across the life course on the association between the metabolic syndrome and CHD risk, and only a small minority have taken account of adult socioeconomic position [1]. Furthermore, several investigators have pointed out that individual components of the syndrome are more important determinants of CHD than either of the WHO or NCEP definitions of the syndrome, thus questioning the clinical utility of the concept of a syndrome for predicting CHD risk [6, 7]. The recent review concluded that more research was needed that specifically 


\section{IDF, Modified WHO and NCEP definitions of metabolic} syndrome used in the analyses

IDF definition of metabolic syndrome.

- Central obesity: waist circumference $\geq 80 \mathrm{~cm}$

- Together with at least two of the following components:

- Raised triglyceride level: $\geq 1.7 \mathrm{mmol} / \mathrm{l}$ OR treatment for this abnormality

- Reduced HDL cholesterol:< $1.29 \mathrm{mmol} / \mathrm{l}$ OR treatment for this abnormality

- Hypertension: raised arterial pressure $\geq 130 / 85$ mm Hg OR antihypertensive medication

- Diabetes: raised fasting plasma glucose $(\geq 5.6 \mathrm{mmol} / \mathrm{l})$ or previously diagnosed type 2 diabetes

Modified WHO definition of metabolic syndrome.

- Clinically diagnosed diabetes OR high fasting glucose (fasting plasma venous glucose $\geq 6.1 \mathrm{mmol} / \mathrm{l}$ ) OR insulin resistance (highest quarter HOMA score)

Together with at least two of the following components:

- Hypertension: raised arterial pressure $\geq 140 / 90$ mm Hg OR antihypertensive medication

- Dyslipidaemia: raised plasma triglycerides $(\geq 1.7 \mathrm{mmol} / \mathrm{l})$ OR low HDL cholesterol $(<1.0 \mathrm{mmol} / \mathrm{l})$

- Central or general obesity: waist to hip ratio $>0.85$ in women OR body mass index $\geq 30 \mathrm{~kg} / \mathrm{m}^{2}$

ATP III definition of metabolic (insulin resistance) syndrome.

Any three (or more) of the following:

- High fasting glucose (fasting plasma venous glucose $\geq 6.1 \mathrm{mmol} / \mathrm{l}$ )

- Hypertension: raised arterial pressure $\geq 130 / 85$ mm Hg OR antihypertensive medication

- Raised plasma triglycerides $(\geq 1.7 \mathrm{mmol} / \mathrm{l})$

- Low HDL cholesterol $(<1.0 \mathrm{mmol} / \mathrm{l})$

- Central obesity (waist circumference $>88 \mathrm{~cm}$ )

addressed the issue of whether any definition of the metabolic syndrome improves risk prediction for adverse events above that of its individual components [1].

In April of this year a new worldwide definition of the metabolic syndrome was proposed by the International Diabetes Federation (IDF) (www.idf.org; text box). This definition was compiled by a panel of experts, including those who had previously been involved in developing the WHO and NCEP definitions. The rationale behind this new classification was to provide a standard definition that could be used across research and clinical groups, and thus enable appropriate comparisons between studies and standardise clinical diagnoses. Further, the new definition was intended to be a better predictor of adverse risk outcomes (specifically, CHD, stroke and type 2 diabetes) than either of the earlier definitions, but to date no study has assessed its performance compared with these earlier definitions.

The aim of this study was to compare the magnitudes of the associations between the new IDF, as well as the WHO and NCEP definitions of the metabolic syndrome, and CHD risk, and to determine whether the magnitudes of the associations for any of the syndrome definitions are greater than those for the individual components.

\section{Subjects, materials and methods}

Full details of the selection of participants and measurements have been reported previously $[2,6,8]$. Women aged 60-79 years were randomly selected from general practitioner lists in 23 British towns. A total of 4,286 women participated and baseline data (self-completed questionnaire, research nurse interview, physical examination and primary-care medical record review) were collected between April 1999 and March 2001. These women have been followed up over a median of 4 years by flagging within the NHS central register for mortality data and by two-yearly review of their medical records. Local ethics committee approvals were obtained for the study and women provided informed written consent for us to access their medical records.

All women with prevalent CHD (doctor diagnosis of previous myocardial infarction, angina or who had a coronary artery bypass or angioplasty; $n=697$ ) at baseline were excluded from this study. Thus, 3,589 women were included in this study. Incident cases of CHD were defined as either (1) CHD death (ICD10 codes I20-I25, I51.6) or (2) a myocardial infarction, diagnosis of angina or coronary artery bypass or angioplasty identified in the follow-up medical record review.

All of the main exposures (different definitions of the metabolic syndrome) and confounders considered in this study were obtained from the baseline assessment. Blood samples were taken after a minimum 6-h fast. Plasma glucose was measured by a glucose oxidase Trinder method [9] using a Hitachi Modular analyser (Hitachi, Kobe, Japan). Serum insulin was measured using an ELISA which does not cross-react with proinsulin [10]. Insulin resistance was estimated according to the homeostasis model assessment (HOMA) as the product of fasting glucose $(\mathrm{mmol} / \mathrm{l})$ and insulin $(\mu \mathrm{U} / \mathrm{ml})$ divided by the constant 22.5 [11]. HDL-cholesterol and triglyceride levels were measured using a Hitachi 747 automated analyser (Hitachi, Tokyo, Japan) and reagents were supplied by Roche Diagnostics (Basel, Switzerland). Standard procedures were used to assess blood pressure, height, weight, and waist and hip circumference as previously described [6]. A clinical diagnosis of type 2 diabetes at baseline was determined from the research interview, assessment of medications and the medical record review [8].

At the research nurse interview all participants brought their current medications and gave details of their drug history. Participants were coded as taking antihypertensive medications if they were currently using medications listed in Section 2.2.1 (Thiazide diuretics), Section 2.2.8 (Diuretics with potassium), Section 2.4 (Beta-blockers), 2.5 (Drugs affecting the renin-angiotensin system and some other antihypertensive drugs), or Section 2.6.2 (Calcium-channel blockers) of the British National Formulary. They were coded as taking medications for the 
treatment of elevated triglycerides or reduced HDL-cholesterol if they were using medications listed in Section 2.12 (lipid-reducing drugs) of the British National Formulary. Of those identified as taking lipid-lowering drugs 93\% were using a statin and $5.9 \%$ were using a fibrate; a small number were taking a mixture of other preparations. Whilst statins are most commonly used for treating high levels of low-density lipoprotein cholesterol, and may not be the treatment of choice for the dyslipidaemia specifically associated with the metabolic syndrome, the new IFD definition includes all cholesterol/dyslipidaemia medications (including statins) in its definition. We did, however, undertake a sensitivity analysis in which we ignored lipid-reducing medications in our definition of the IFD syndrome.

The text box shows the IDF, WHO and NCEP definitions of the metabolic syndrome used in this study. We used a modified version of the WHO definition of the syndrome as proposed for use in epidemiological studies by the European Group for the study of Insulin Resistance [12]. This differs from the proposed WHO definition [13] in three ways - high fasting glucose $(\geq 6.1 \mathrm{mmol} / \mathrm{l})$ is used in place of glucose intolerance assessed by oral glucose tolerance test, HOMA scores are used to assess insulin resistance instead of euglycaemic clamp techniques, and microalbuminuria is not included in the definition.

Data on socioeconomic indicators across the life-course were obtained from baseline questionnaires and included data on the longest held occupation of the participant's father during her childhood, childhood household amenities (bathroom, hot water, bedroom sharing and car access), age at completion of full-time education, the longest held occupation of the participant and her spouse, adult housing tenure, car access, and pension arrangements. Childhood social class of the women was based on their fathers' longest held occupation and adult social class was based on their husbands' longest held occupation, or their own, whichever was higher. Adult and childhood social class were defined according to the registrar general's classification of occupations (I, II, III non-manual, III manual, IV, $\mathrm{V}$-with I being professional occupations and $\mathrm{V}$ being manual unskilled occupations). Most of the indicators of socioeconomic position were binary variables. For some analyses we dichotomised those indicators that were not binary as follows: adult and childhood social class into non-manual (I, II, III non-manual) and manual (III manual, IV, V); pension arrangements into state only or state plus other (employment or private pension); adult housing tenure into local authority (social housing) or other (owner occupied, private rental, living with a relative) and age at leaving full-time education into those leaving school at or below the minimum legal age for leaving education or above that age. Data on smoking (classified as never, past, current - including those who said they had quit smoking in the 6-month period prior to assessment) and physical activity (categorised as $\leq 2,2-3, \geq 3 \mathrm{~h}$ per week of either moderate or vigorous activity) were obtained from the interview or questionnaires.

\section{Statistical analyses}

Data were analysed using Cox proportional hazards regression models, with participants' age as the time axis and the date of the baseline examination as the beginning of the follow-up period. This means that all analyses have been adjusted for age. Contributions to risk were censored at the earlier of: (1) first episode of either a fatal or non-fatal CHD event (if an individual had repeated non-fatal CHD events they were censored at the first event); (2) death from a cause other than CHD; (3) 30 November 2004. Proportionality assumptions were assessed by inspection of cumulative incidence plots and by testing for evidence of a statistical interaction with the time-scale of the models. There was no evidence of any violation of the proportionality assumption in any models.

For several of the covariates there were small amounts of missing data, the greatest amount of missing data being for pension arrangements $(368[10 \%]$ of the 3,589 women included in this analysis did not answer this question). We used multiple multivariate imputation, using all other covariates, the outcome, the log of survival time and the censoring indicator, to impute a distribution of missing values for those variables with some missing data with switching regression [14]. We carried out 20 cycles of regression switching and generated five imputation datasets, and also undertook all analyses on the complete dataset subsample $(n=2,807$ [78\%]). The results from the complete data subset analyses were essentially the same as those presented here but were less precisely estimated.

For individual components of the metabolic syndrome, we assessed whether or not associations were linear by splitting the variables into fifths and computing a likelihood ratio test that compared a model in which the fifths were entered as four indicator variables and one in which they were entered as a continuous score from 1 to 5 . This likelihood ratio test (referred to in tables as $p$ value for nonlinear associations) can then be interpreted as assessing the null hypothesis that any non-linear association is a 'better' model than a linear association.

Whilst all three definitions include high fasting glucose as a component, only the WHO criteria include a clinical diagnosis of diabetes as part of the syndrome. Studies that have assessed the association of the syndrome (defined by whatever means) with CHD have varied with respect to whether they include participants with diagnosed diabetes, exclude these participants, or do not discuss how patients with diabetes are categorised. For the main analyses presented here we did not exclude women with clinical diagnoses of diabetes. For the two definitions that do not explicitly include participants with diabetes (IFD, NCEP) we retained the definitions as published and so any woman with a clinical diagnosis but who had a blood glucose below $6.1 \mathrm{mmol} / 1$ would not be defined as having high blood glucose ( $n=39$ out of 157 with diabetes). We then repeated all analyses with women who had diabetes $(n=157$ [4.4\%] of the 3,589 women included in this analysis) excluded. 
Since comorbidities that might lead to weight reduction and changes in metabolic risk factors are more likely at older age, these metabolic risk factors might predict CHD less well in older individuals. We therefore explored whether the effects varied by age group (60-69 years versus 70-79 years at baseline) or by self-reported health status at baseline (good or excellent versus poor or fair health at present). We computed likelihood ratio tests to determine whether there was statistical evidence of a difference by age group or health status. Robust standard errors, taking account of possible non-independence between individuals from the same town, were used to estimate $p$ values and 95\% confidence intervals. All analyses were conducted using Stata version 8.0 (StataCorp, College Station, TX, USA).

Table 1 Baseline characteristics among British women aged 60-79 years who were free of coronary heart disease $(n=3,589)$

Values are mean (SD), number $(\%)$ or geometric means $(95 \%$ (Is)*

HOMA homeostasis model assessment of insulin resistance, IDF International Federation of Diabetes, $W H O$ World Health Organization, NCEP National Cholesterol Education Programme, HDLc HDL-cholesterol, $B P$ blood pressure $T G$ triglycerides

\section{Results}

Over the follow-up period there were 194 (40 fatal) incident cases of CHD during 15,778 woman-years of follow-up giving a rate of 12.3 (95\% CI: $10.7,14.2)$ per 1,000 woman-years. Table 1 shows baseline characteristics of the 3,589 women included in this analysis. The prevalence of the metabolic syndrome varied by each definition, being $48 \%$ with the IDF, $21 \%$ with WHO and $29 \%$ with NCEP. These differences were largely driven by the lower thresholds for defining central obesity and high blood pressure in the IDF definition.

Table 2 shows the unadjusted associations between both the metabolic syndrome and each component of the syndrome and CHD. Insulin resistance (HOMA score), tri-

\begin{tabular}{|c|c|}
\hline Characteristic & Values \\
\hline HOMA score* & $1.60(1.57,1.63)$ \\
\hline Fasting glucose $(\mathrm{mmol} / \mathrm{l})^{*}$ & $5.89(5.86,5.93)$ \\
\hline Triglyceride $(\mathrm{mmol} / \mathrm{l})^{*}$ & $1.65(1.62,1.68)$ \\
\hline $\operatorname{HDLc}(\mathrm{mmol} / \mathrm{l})$ & $1.66(0.45)$ \\
\hline Systolic blood pressure (mm Hg) & $147.7(24.9)$ \\
\hline Diastolic blood pressure (mm Hg) & $79.7(11.6)$ \\
\hline Body mass index $\left(\mathrm{kg} / \mathrm{m}^{2}\right)$ & $27.4(4.9)$ \\
\hline Waist-to-hip ratio & $0.817(0.068)$ \\
\hline Waist circumference (mm) & $857.5(119.9)$ \\
\hline IDF metabolic syndrome & $1,707(47.5 \%)$ \\
\hline IDF central obesity $(\geq 80 \mathrm{~cm})$ & $2,358(65.7 \%)$ \\
\hline IDF raised triglycerides $(\geq 1.7 \mathrm{mmol} / 1$ or on treatment $)$ & $1,700(47.3 \%)$ \\
\hline IDF reduced HDLc $(<1.29 \mathrm{mmol} / 1$ or on treatment $)$ & $751(20.9 \%)$ \\
\hline IDF hypertension $(\mathrm{BP} \geq 130 / 85$ or on treatment $)$ & $2,850(79.4 \%)$ \\
\hline IDF diabetes (fasting glucose $\geq 5.6 \mathrm{mmol} / 1$ or type 2 diabetes) & $1,870(52.1 \%)$ \\
\hline WHO metabolic syndrome & $749(20.9 \%)$ \\
\hline WHO insulin resistance (top quarter HOMA in non-diabetics $\geq 2.46$ ) & $906(25.2 \%)$ \\
\hline WHO diabetes (fasting glucose $>6.1 \mathrm{mmol} / 1$ or type 2 diabetes) & $816(22.7 \%)$ \\
\hline WHO dyslipidaemia $(\mathrm{TG} \geq 1.7$ or HDLc $<1.0 \mathrm{mmol} / \mathrm{l}$ ) & $1,643(45.8 \%)$ \\
\hline WHO hypertension (BP $\geq 140 / 90$ or on treatment) & $2,460(68.5 \%)$ \\
\hline WHO obesity $(\mathrm{BMI} \geq 30.0$ or $\mathrm{WHR}>0.85)$ & $1,556(43.4 \%)$ \\
\hline NCEP metabolic syndrome & $1,071(29.8 \%)$ \\
\hline NCEP high fasting glucose $(>6.1 \mathrm{mmol} / \mathrm{l})$ & $898(25.0 \%)$ \\
\hline NCEP hypertension (BP $\geq 130 / 85$ or on treatment) & $2,850(79.4 \%)$ \\
\hline NCEP low HDLc $($ HDLc $<1.0 \mathrm{mmol} / \mathrm{l})$ & $101(2.8 \%)$ \\
\hline NCEP high TG $(\mathrm{TG} \geq 1.7 \mathrm{mmol} / \mathrm{l})$ & $1,542(43.0 \%)$ \\
\hline NCEP obesity (waist circumference $>880 \mathrm{~mm}$ ) & $1,450(40.4 \%)$ \\
\hline Childhood manual social class & $2,742(76.4 \%)$ \\
\hline Childhood no bathroom in house & $1,372(38.2 \%)$ \\
\hline Childhood no hot water in house & $1,241(34.6 \%)$ \\
\hline Childhood shared bedroom & $1,869(52.1 \%)$ \\
\hline Childhood no car access & $2,924(81.5 \%)$ \\
\hline Left full-time education at minimum legal age & $1,240(34.6 \%)$ \\
\hline Adult manual social class & $1,873(52.2 \%)$ \\
\hline Adult living in social housing & $448(12.5 \%)$ \\
\hline Adult no car access & $989(27.6 \%)$ \\
\hline Adult state pension arrangements only & $989(27.6 \%)$ \\
\hline Current smoker & $422(11.8 \%)$ \\
\hline Physically inactive ( $<2 \mathrm{~h}$ per week moderate/vigorous activity) & $634(17.7 \%)$ \\
\hline
\end{tabular}


glyceride levels, systolic blood pressure and waist-to-hip ratio were all positively and linearly associated with CHD risk in these unadjusted analyses, and HDL cholesterol was inversely associated with CHD risk. Body mass index was not associated with CHD risk. All three definitions of the metabolic syndrome were positively associated with CHD risk. The magnitudes of all three associations were modest and similar. The magnitudes of the associations for individual components of the syndrome were similar to or larger than those for the any of the syndrome definitions. In particular, the point estimates for the IDF (and NCEP which uses the same criteria) definition of hypertension and NCEP definition of low HDL cholesterol were stronger than those for the syndrome defined by any of the three definitions (though there was no statistical evidence that the estimates for any individual components were different to those for the syndrome as a whole, all $p$ values being $<0.3)$.
Table 3 shows the effect of adjustment for potential confounding factors on these associations. For the components of the metabolic syndrome these have been included as continuous variables in these multivariable analyses since their associations in unadjusted analyses were linear across their distribution. Adjustment for smoking and physical inactivity resulted in some attenuation of the linear associations of insulin resistance, triglycerides, HDL cholesterol and waist-to-hip ratio with CHD risk, but greater attenuation occurred with adjustment for indicators of socioeconomic position from across the life-course. In the fully adjusted models each standard deviation increase in HOMA score was associated with a $17 \%$ increased hazard of CHD, with a similar magnitude of positive association with triglyceride levels. In the fully adjusted model each standard deviation of HDL cholesterol was associated with a $24 \%$ decrease in CHD hazard. The association between waist-to-hip ratio and CHD indicated a 10\% increased risk

Table 2 Age-adjusted associations between metabolic syndrome and components of the metabolic syndrome, and incident CHD ( $n=3,589)$

\begin{tabular}{|c|c|c|c|}
\hline Exposure & HR $(95 \% \mathrm{CI})$ & $\begin{array}{l}p \text { linear } \\
\text { trend }\end{array}$ & $\begin{array}{l}p \text { nonlinear } \\
\text { association }^{\mathrm{a}}\end{array}$ \\
\hline \multicolumn{4}{|l|}{ Components as continuous exposures } \\
\hline HOMA score (per SD) & $1.23(1.10,1.38)$ & $<0.001$ & 0.6 \\
\hline Fasting glucose (per SD) & $1.10(0.97,1.26)$ & 0.09 & 0.3 \\
\hline Triglyceride level (per SD) & $1.25(1.12,1.40)$ & $<0.001$ & 0.2 \\
\hline HDLc (per SD) & $0.71(0.61,0.82)$ & $<0.001$ & 0.9 \\
\hline Systolic blood pressure (per SD) & $1.18(1.01,1.37)$ & 0.01 & 0.08 \\
\hline Diastolic blood pressure (per SD) & $1.06(0.94,1.20)$ & 0.3 & 0.08 \\
\hline Body mass index (per SD) & $1.03(0.89,1.19)$ & 0.7 & 0.7 \\
\hline Waist-to-hip ratio (per SD) & $1.15(1.02,1.31)$ & 0.02 & 0.005 \\
\hline Waist circumference (per SD) & $1.12(0.98,1.27)$ & 0.09 & 0.6 \\
\hline \multicolumn{4}{|c|}{ IDF definite metabolic syndrome and IDF-defined dichotomous components } \\
\hline IDF metabolic syndrome & $1.32(1.03,1.70)$ & & \\
\hline IDF central obesity $(\geq 80 \mathrm{~cm})$ & $1.24(0.96,1.59)$ & & \\
\hline IDF raised triglycerides $(\geq 1.7 \mathrm{mmol} / \mathrm{l}$ or on treatment $)$ & $1.48(1.13,1.94)$ & & \\
\hline IDF reduced HDLc $(<1.29 \mathrm{mmol} / 1$ or on treatment $)$ & $1.26(0.88,1.80)$ & & \\
\hline IDF hypertension (BP $\geq 130 / 85$ or on treatment) & $2.09(1.14,3.74)$ & & \\
\hline IDF diabetes (fasting glucose $\geq 5.6 \mathrm{mmol} / 1$ or type 2 diabetes) & $1.22(0.96,1.54)$ & & \\
\hline \multicolumn{4}{|c|}{ WHO definite metabolic syndrome and WHO-defined dichotomous components } \\
\hline WHO metabolic syndrome & $1.45(1.00,2.10)$ & & \\
\hline WHO Insulin resistance (top quarter HOMA in non-diabetics $\geq 2.46$ ) & $1.43(0.99,2.07)$ & & \\
\hline WHO Diabetes (fasting glucose $>6.1 \mathrm{mmol} / 1$ or type 2 diabetes) & $1.21(0.72,2.05)$ & & \\
\hline WHO Dyslipidaemia (TG $\geq 1.7$ or HDLc $<1.0 \mathrm{mmol} / \mathrm{l})$ & $1.53(1.18,1.97)$ & & \\
\hline WHO Hypertension ( $\mathrm{BP} \geq 140 / 90$ or treatment) & $1.67(1.08,2.54)$ & & \\
\hline WHO Obesity (BMI $\geq 30.0$ or WHR $>0.85$ ) & $1.31(1.07,1.61)$ & & \\
\hline \multicolumn{4}{|c|}{ NCEP definite metabolic syndrome and NCEP-defined dichotomous components } \\
\hline NCEP metabolic syndrome & $1.38(1.00,1.93)$ & & \\
\hline NCEP High fasting glucose $(>6.1 \mathrm{mmol} / \mathrm{l})$ & $1.25(0.82,1.89)$ & & \\
\hline NCEP Hypertension (BP $\geq 130 / 85$ or medication) & $2.09(1.14,3.74)$ & & \\
\hline NCEP Low HDLc $($ HDLc $<1.0 \mathrm{mmol} / \mathrm{l})$ & $2.01(0.95,4.26)$ & & \\
\hline NCEP High TG $(\mathrm{TG} \geq 1.7 \mathrm{mmol} / \mathrm{l})$ & $1.60(1.25,2.08)$ & & \\
\hline NCEP Obesity (waist circumference $>880 \mathrm{~mm}$ ) & $1.32(1.04,1.68)$ & & \\
\hline
\end{tabular}

HOMA homeostasis model assessment of insulin resistance, $W H O$ World Health Organization, $H D L c$ HDL-cholesterol, $B P$ blood pressure $T G$ triglycerides, ATP III third adult treatment panel

${ }^{\text {a }}$ Likelihood ratio test comparing a model with fifths of the distribution of each variable entered as four indicator variables to one with the fifths entered as a continuous score from 1 to 5 
Table 3 Multivariable analyses of components of metabolic syndrome and three definitions of the metabolic syndrome with incident coronary heart disease $(n=3,589)$

$$
\text { Age-adjusted }
$$

\author{
Smoking and
}

inactive adjusted
Smoking, inactive and life-course Smoking, inactive, life-course

socioeconomic position* adjusted socioeconomic position* and other metabolic component ${ }^{* *}$ adjusted

\begin{tabular}{llll}
\hline HOMA (per SD) & $1.23(1.10,1.38)$ & $1.21(1.08,1.36)$ & $1.17(1.04,1.31)$ \\
Triglycerides (per SD) & $1.25(1.12,1.40)$ & $1.21(1.09,1.35)$ & $1.17(1.04,1.32)$ \\
HDLc (per SD) & $0.71(0.61,0.82)$ & $0.73(0.63,0.85)$ & $0.76(0.64,0.89)$ \\
Systolic BP (per SD) & $1.18(1.01,1.37)$ & $1.18(1.01,1.37)$ & $1.17(1.01,1.37)$ \\
Waist-to-hip ratio (per SD) & $1.15(1.02,1.31)$ & $1.12(0.98,1.27)$ & $1.10(0.96,1.26)$ \\
Metabolic syndrome & & & \\
$\quad$ IDF-defined & $1.32(1.03,1.70)$ & $1.29(1.00,1.66)$ & $1.25(0.96,1.61)$ \\
WHO-defined & $1.45(1.00,2.10)$ & $1.39(0.96,2.01)$ & $1.31(0.90,1.90)$ \\
NCEP-defined & $1.38(1.00,1.93)$ & $1.34(0.96,1.86)$ & $1.27(0.90,1.79)$
\end{tabular}

$0.99(0.80,1.22)$

$1.02(0.84,1.22)$

$0.76(0.61,0.96)$

$1.16(1.00,1.35)$

$1.03(0.89,1.19)$

HOMA homeostasis model assessment of insulin resistance, $I D F$ International Diabetes Federation, WHO World Health Organization, NCEP National Cholesterol Education Programme, HDLc HDL-cholesterol, BP blood pressure, TG triglycerides

*Life-course socioeconomic position: adjusted for childhood social class, bathroom in childhood home, hot water in childhood home, shared bedroom in childhood, family access to a car in childhood, age at leaving full-time education, adult social class, housing type in adulthood, car access in adulthood, pension arrangements for retirement

**A model including simultaneously all five of the continuous metabolic traits as well as all other covariates. These results should be treated with caution since there is evidence in this model of co-linearity between the metabolic syndrome traits

of CHD for each standard deviation with the lower $95 \%$ confidence limit being just below the null value in the fully adjusted model. The association between systolic blood pressure and CHD risk was not affected by adjustment for smoking, physical activity or life-course socioeconomic position. As would be anticipated with the effect of these adjustments on individual components of the syndrome, the associations between all three definitions of the syndrome and CHD attenuated towards the null with adjustment for life-course socioeconomic position. In the fully adjusted model each definition was associated with an approximate $30 \%$ increase in CHD risk.

When all components of the metabolic syndrome were included together in the same model along with potential confounding factors, only HDL cholesterol and systolic blood pressure remained independently associated with CHD risk (final column in Table 3). However, there was evidence of co-linearity in this model between the components of the metabolic syndrome, with the standard errors for the logged hazard ratio for each of HOMA score, triglyceride levels, systolic blood pressure and waist-to-hip ratio increasing considerably in this model compared with models with just one component included. The effects of components of the metabolic syndrome and of each definition of the syndrome did not vary by age group or by health status at baseline of the women (all $p$ values for interaction $>0.2$ ). When women on lipid-reducing medications were ignored in our IFD definition of the syndrome, the prevalence of this syndrome was reduced very slightly from 47.5 to $47.3 \%$, and none of the point estimates of associations in age-adjusted or multivariable associations changed. When we repeated the analyses with those 157 $(4.4 \%)$ women who had a clinical diagnosis of diabetes removed, the findings were essentially the same as those presented for the main analyses. With these women removed, there were 185 incident cases of CHD over 15,076 years of follow-up, giving a rate of 12.3 (95\%CI: 10.6 , 14.2) per 1,000 person-years that was the same as that for the whole cohort. The age-adjusted hazard ratios of CHD for each syndrome in those without diabetes at baseline were $1.49(0.99,2.24)$ for the WHO syndrome, 1.44 (1.03, $2.01)$ for the NCEP syndrome and $1.37(1.06,1.77)$ for the IFD syndrome. With further adjustment for life-course socioeconomic position, smoking and physical inactivity these attenuated to $1.34(0.88,2.04), 1.31(0.92,1.89)$ and $1.28(0.98,1.69)$, respectively.

To indicate the importance of the metabolic syndrome as a risk factor for CHD in postmenopausal women, we compared its effect with that of smoking. The hazard ratio of being an ex-smoker compared to a never smoker was 1.36 (95\%CI: $0.97,1.93)$ and of being a current smoker compared to a never smoker was 2.54 (95\%CI: 1.64, 3.94) in the unadjusted models. With adjustment for physical inactivity, life-course socioeconomic position and components of the metabolic syndrome, these associations were only slightly attenuated: 1.31 (95\%CI: $0.92,1.89) \mathrm{com}-$ paring ex-smokers to never smokers and 2.20 (5\%CI: 1.41 , 3.42 ) comparing current smokers to never smokers.

\section{Discussion}

In a large prospective study of women we found insulin resistance, triglyceride levels, systolic blood pressure and central adiposity to be linearly and positively associated with CHD risk. We also found that HDL cholesterol was linearly inversely associated with CHD risk. Upon adjustment for smoking, physical activity and life-course socioeconomic position these linear associations were attenuated, but nevertheless remained. The metabolic syndrome as defined using IDF, WHO or NCEP criteria was only moderately associated with increased CHD risk, with 
these associations attenuating towards the null upon adjustment for life-course socioeconomic position. Furthermore, the magnitudes of associations for individual components of the syndrome were similar to or larger than for the syndrome, questioning the clinical or predictive utility of defining the syndrome with respect to a risk factor for CHD. Of note, the point estimates for NCEP-defined hypertension and low HDL-cholesterol suggested a doubling ( $200 \%$ increase) of risk, whereas the point estimate for the syndrome suggested an increased risk of just 30$40 \%$. This will occur because some women with isolated hypertension or HDL cholesterol or just these and one other risk factor will not be defined as having the syndrome. Thus from a clinical point of view some women with increased risk may not be identified as such if the metabolic syndrome is used as a major way of defining cardiovascular disease risk. There was no suggestion that the new IDF worldwide definition predicted CHD risk with greater magnitude than either of the WHO or NCEP definitions. In contrast to the findings for the metabolic syndrome, current smoking showed a much stronger association with CHD risk in unadjusted analyses, with very little attenuation of this effect upon adjustment for life-course socioeconomic position and components of the metabolic syndrome.

\section{Study limitations}

We had just one baseline measurement of each exposure variable and therefore our effect estimates could be an underestimation of the true association if regression dilution bias were taken into account. The new IFD definition of the syndrome includes, in the dyslipidaemia component, those women who are taking medication for this condition. We included here any woman on a drug defined in the British National Formulary as a lipid-lowering drug. The majority were taking a statin, which is a type of drug largely prescribed for high levels of total or of low-density lipoprotein cholesterol and we do not have clinical information on exactly what form of dyslipidaemia each woman on these medications had. However, when we repeated the analyses ignoring lipid-lowering treatment, the results were not affected. We used retrospective report in adulthood of childhood socioeconomic circumstances and these may be inaccurate. A recent study of Scottish individuals in their early 50 s found modest agreement between adult retrospective report of childhood socioeconomic position and information collected in childhood. However, the magnitude of the association of retrospective report with CHD risk and that of the association of collected-inchildhood socioeconomic position data with CHD risk was the same in that cohort [5].

Is the metabolic syndrome an important risk factor for CHD in women?

Our results suggest linear associations between insulin resistance (and its associated metabolic traits) and CHD risk that, although attenuated, persist after adjustment for lifecourse socioeconomic position. However, the magnitude of effect of the metabolic syndrome defined using any of the three definitions was similar to or weaker than the magnitude of effect of many of the individual components (see Table 2), suggesting that in clinical and research practice there is little prognostic value in defining the components as a 'syndrome' for the prediction of CHD. The point estimates of effect for each syndrome found in this study are somewhat weaker than those found in a recent metaanalysis (1.7 to 1.9$)$ of the associations of WHO-defined and NCEP-defined syndromes with cardiovascular disease mortality [1]. However, examination of 95\% CIs would suggest that our estimates are in fact consistent with those from that meta-analysis. None of the studies included in that meta-analysis had performed an adjustment for socioeconomic position from across the life-course and only a minority had taken adult socioeconomic position into account. The author of that review concluded that the metabolic syndrome was not an important predictor of allcause mortality or of cardiovascular disease in general population studies, although it may turn out to be important in some population subgroups.

Our findings are in women only and in an older age group and it is possible that the syndrome is a stronger predictor of disease outcomes in younger age groups and in men. However, our findings are consistent with those found in the West of Scotland Coronary Prevention Study (WOSCOP), which examined younger men and is one of the biggest studies to date to assess the association of the metabolic syndrome with CHD risk [15]. Furthermore, the relative risks of CHD associated with diabetes are greater in women than men $[16,17]$, and since the metabolic syndrome is considered to be a precursor of type 2 diabetes one might expect stronger associations of the metabolic syndrome with CHD in women compared to men.

There was some suggestion from mutual adjustment for each component that HDL cholesterol and systolic blood pressure were the most important individual risk factors (being independent of other metabolic risk factors). However, the components of the syndrome are associated with each other with evidence of co-linearity in the model containing all components and therefore there findings should be treated with caution.

One possible explanation for the syndrome not being a strong predictor of CHD, despite strong linear associations between the individual components and CHD, is that the syndrome inevitably represents a heterogeneous group with the overall effect estimate being a weighted average of some very high-risk individuals and some who are lower risk. For example, low HDL cholesterol and hypertension were particularly strong predictors of CHD in this population of older women, but many women defined as having the syndrome may not have either of these risk factors. In all three definitions, each component is dichotomised, despite having linear associations with CHD. In the most commonly used risk prediction method for CHD, the Framingham risk equation [18], continuous variables are not dichotomised and a continuum of risk is derived 
rather than a dichotomy that groups together a heterogeneous population. However, the Framingham equation has been found to be a poor predictor of CHD risk in a number of groups, including those with diabetes [19], women [20] and older individuals [21]. It may be that in the population as a whole, and in these specific groups, that adding some components of the metabolic syndrome and other novel risk factors to the Framingham equation will improve its predictive ability.

As the ability to test and compare different predictive models in different population groups increases along with the use of computers in clinical practice, it should become possible for more accurate prediction of risk in the clinical setting with easy to use computer programs. These programs should also be capable (behind the scenes) of taking account of effect modification (for example differences in effect by sex, age, ethnicity) of risk factors and of modelling each risk factor in a way (linear, quadratic, cubic) that best reflects its association with CHD. In the mean time researchers should be cautious of promoting syndrome definitions as important risk factors for disease outcomes, when these syndromes may mask important heterogeneity within the group defined as having the syndrome.

The magnitude of effect of the metabolic syndrome in this cohort of postmenopausal women was similar to that of being a past smoker compared to a never smoker, but weaker than the effect of being a current smoker. Thus, whilst individual components of the syndrome, notably insulin resistance, triglyceride levels, HDL cholesterol and systolic blood pressure, are linearly related to CHD risk even after adjustment for life-course socioeconomic position, smoking and physical inactivity, the syndrome itself is only weakly associated with CHD risk and does not appear to be a specific risk factor for CHD in postmenopausal women. Our findings, together with those of other prospective studies [1], question the importance of the metabolic syndrome per se as a risk factor for CHD.

Acknowledgements The British Women's Heart and Health Study is codirected by Peter Whincup and Goya Wannamethee in addition to Shah Ebrahim and Debbie Lawlor. We thank Rita Patel, Carol Bedford, Alison Emerton, Nicola Frecknall, Karen Jones, Mark Taylor, Simone Watson and Katherine Wornell for collecting and entering data; all of the general practitioners and their staff who have supported data collection; and the women who have participated in the study. We thank the (UK) Department of Health for core support to the British Women's Heart \& Health Study and the British Heart Foundation for additional funding. Debbie A. Lawlor is funded by a (UK) Department of Health career scientist award. The views expressed in this paper are those of the authors and not necessarily those of any funding body. No funding body has influenced the analysis or its interpretation.

\section{References}

1. Ford ES (2005). Risks for all-cause mortality, cardiovascular disease, and diabetes associated with the metabolic syndrome: a summary of the evidence. Diabetes Care 28:1769-1778
2. Lawlor DA, Ebrahim S, Davey Smith G (2002) Socioeconomic position in childhood and adulthood and insulin resistance: cross sectional survey using data from the British Women's Heart and Health Study. BMJ 325:805-807

3. Parker L, Lamont DW, Unwin N et al (2003). A lifecourse study of risk for hyperinsulinaemia, dyslipidaemia and obesity (the central metabolic syndrome) at age 49-51 years. Diabet Med 20:406-415

4. Lawlor DA, Ebrahim S, Davey Smith G (2002) A lifecourse approach to coronary heart disease and stroke. In: Kuh D, Hardy R (eds) A life course approach to women's health, Oxford University Press, Oxford, pp 86-120

5. Lawlor DA, Ronalds G, Macintyre S, Clark H, Leon DA (2005) Father's occupational social class at the time of birth predicts future cardiovascular disease risk: findings from the Aberdeen Children of the 1950s cohort study. Am J Public Health (in press)

6. Lawlor DA, Ebrahim S, Davey Smith G (2004) The metabolic syndrome and coronary heart disease in older women: findings from the British Women's Heart and Health Study. Diabet Med 21:906-913

7. Tanko LB, Bagger YZ, Qin G, Alexandersen P, Larsen PJ, Christiansen C (2005) Enlarged waist combined with elevated triglycerides is a strong predictor of accelerated atherogenesis and related cardiovascular mortality in postmenopausal women. Circulation 111:1883-1890

8. Lawlor DA, Ebrahim S, Davey SG (2002). The association between components of adult height and Type II diabetes and insulin resistance: British Women's Heart and Health Study. Diabetologia 45:1097-1106

9. Trinder P (1969) Determination of blood glucose using 4-amino phenaz as oxygen acceptor. J Clin Pathol 22:246

10. Andersen L, Dinesen B, Jorgensen PN, Poulsen F, Roder ME (1993) Enzyme immunoassay for intact human insulin in serum or plasma. Clin Chem 39:578-582

11. Matthews DR, Hosker JP, Rudenski AS, Naylor BA, Treacher DF, Turner RC (1985) Homeostasis model assessment: insulin resistance and beta-cell function from fasting plasma glucose and insulin concentrations in man. Diabetologia 28:412-419

12. Balkau B, Charles MA (1999) Comment on the provisional report from the WHO consultation. European Group for the Study of Insulin Resistance (EGIR). Diabet Med 16:442-443

13. World Health Organization (1999) Definition, diagnosis and classification of diabetes mellitus and its complications. Report of a WHO consultation. Part 1: Diagnosis and classification of diabetes mellitus, Geneva: WHO,1999

14. Royston P (2004) Multiple imputation of missing values. Stata Journal 4:227-241

15. Sattar N, Gaw A, Scherbakova O et al (2003) Metabolic syndrome with and without C-reactive protein as a predictor of coronary heart disease and diabetes in the West of Scotland Coronary Prevention Study. Circulation 108:414-419

16. Lee WL, Cheung AM, Cape D, Zinman B (2000) Impact of diabetes on coronary artery disease in women and men: a metaanalysis of prospective studies. Diabetes Care 23:962-968

17. Yusuf S, Hawken S, Ounpuu S et al (2004) Effect of potentially modifiable risk factors associated with myocardial infarction in 52 countries (the INTERHEART study): casecontrol study. Lancet 364:937-952

18. Anderson KM, Odell PM, Wilson PW, Kannel WB (1990) Cardiovascular disease risk profiles. Am Heart J 121:293-298

19. McEwan P, Williams JE, Griffiths A et al (2004) Evaluating the performance of the Framingham risk equations in a population with diabetes. Diabet Med 21:318-323

20. Michos ED, Nasir K, Braunstein JB et al (2005) Framingham risk equation underestimates subclinical atherosclerosis risk in asymptomatic women. Atherosclerosis DOI:10.1016/j. atherosclerosis.2005.04.004

21. Brindle P, Emberson J, Lampe F et al (2003) Predictive accuracy of the Framingham coronary risk score in British men: prospective cohort study. BMJ 327:1267 